Research Article

Delin Sun* and Minggao Zhu

\title{
Effect of Pressure Distribution on the Energy Dissipation of Lap Joints under Equal Pre-tension Force
}

https://doi.org/10.1515/phys-2019-0034

Received Mar 27, 2019; accepted Apr 18, 2019

Abstract: In this paper, the energy dissipation in a bolted lap joint is studied using a continuum microslip model. Five contact pressure distributions compliant with the power law are considered, and all of them have equal pretension forces. The effects of different pressure distributions on the interface stick-slip transitions and hysteretic characteristics are presented. The calculation formulation of the energy dissipation is introduced. The energy dissipation results are plotted on linear and log-log coordinates to investigate the effect of the pressure distribution on the energy distribution. It is shown that the energy dissipations of the lap joints are related to the minimum pressure in the overlapped area, the size of the contact area and the value of the power exponent. The work provides a theoretical basis for further effective use of the joint energy dissipation.

Keywords: Bolted joints, Pretention force, Pressure distribution, Energy dissipation

PACS: 05.45.-a, 61.72.Hh

\section{Introduction}

Many engineering structures consist of multiple components that are fastened by bolts. The bolted joints have notable effects on the structures' responses. In dynamic environments, bolted joints are the main source of damping, and contribute to $90 \%$ of the energy dissipation of jointed structures [1, 2]. Meanwhile, joint interfaces present very complicated, non-linear behaviors that vary with load amplitudes, which result in non-linear responses $[3,4]$.

\footnotetext{
*Corresponding Author: Delin Sun: Ulsan Ship and Ocean Col-
lege Ludong University, Yantai, Shandong 264025, China; Email

^Corresponding Author: Delin Sun: Ulsan Ship and Ocean Col-
lege Ludong University, Yantai, Shandong 264025, China; Email: sundelin@ldu.edu.cn

Minggao Zhu: Department of Physics, Clark Atlanta University, Atlanta, GA 30314, United States of America
}

¿ Open Access. @ 2019 D. Sun and M. Zhu, published by De Gruyter. 4.0 License
Microslip is the main mechanism of the joint energy dissipation and non-linearity $[5,6]$. According to experimental studies, the main factors affecting microslip and energy dissipation are normal loads, components materials, surface roughness and geometry dimensions of the joints (bolts, washers and components), etc. [7-11]. The normal load is usually characterized as the bolt pretension force. As is well known, bolt pre-tension force is negatively correlated with joint energy dissipation.

The pre-tension forces, components materials and geometry dimensions directly result in different interface pressure distributions [12]. Hence, use of the interface pressure distribution to characterize the normal load is more specific than any of these other factors. Significant effort has been expended in studying the relationship between interface pressure distribution and microslip. Menq analyzed the dynamic responses of a damper under a uniform pressure distribution [13]. Casba presented responses of a damper subjected to normal tractions with quadrature pressure distribution [14]. Song et al. studied a lap joint subjected to power- and Gaussian-function pressure distributions, and presented the power-law relationship between the energy dissipation and tangential forces [15]. Cigeroglu considered the effect of inertia of elastic rods and analyzed stick-slip transitions of the contact interfaces under convex, concave and uniform pressure distributions [16]. Xiao et al. analyzed the effect of pressure distributions with four different power-function exponents on energy dissipation of the lap joints [17].

Of the literature mentioned above, most of the studies emphasize the effect of pressure distribution compared with different types of functions of the energy dissipation. Xiao considered the distributions of power function with different exponents, in which the contact area and minimum pressure are assumed to be equal. So, in essence, Xiao's research is more inclined to be about the effect of pre-tension force on the energy dissipation of the lap joint. It is of engineering benefits to study the effect of different pressure distributions on the energy dissipation under equal bolt pre-tention force, which helps further compre- 
hend the mechanism of energy dissipation and effectively use energy dissipation to reduce the structure responses.

The purpose of this work is to evaluate the effect of the pressure distribution on the energy dissipation of bolted joints. A continuum model is employed to simulate the lap joint. Five power functions with different coefficients and exponents, to express different distribution characteristics, are considered. The static response of a microslip model is obtained, then the effects of pressure distributions on the interface stick-slip transitions, hysteretic characteristics and energy dissipation are analyzed in successive sections, leading to a brief conclusion.

\section{Bolted lap joint microslip model}

The study object is a shear lap joint under a longitudinal force, as shown in Figure 1. The length of the overlap zone is $2 L$. The upper and lower parts are approximated to elastic rods incapable of supporting bending moments. For convenience, the structure is simplified as the continuum model shown in Figure 2.

In this model, only the right half of the lap joint is considered. The model contains an elastic rod and a rigid base with a shear layer of negligible thickness between them. The elastic rod has a length of $L$, a uniform Young's modulus $E$ and a constant cross-sectional area $A . \tau$ denotes the stress on the shear layer, which has property similar to ideal elastic-plastic materials, as shown in Figure 3. $u$

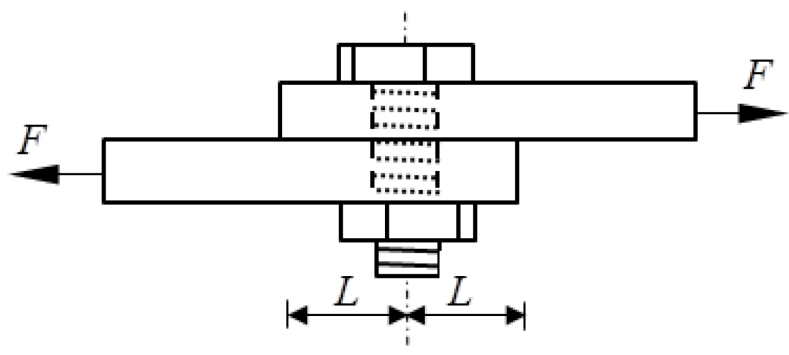

Figure 1: Sketch of the lap joint.

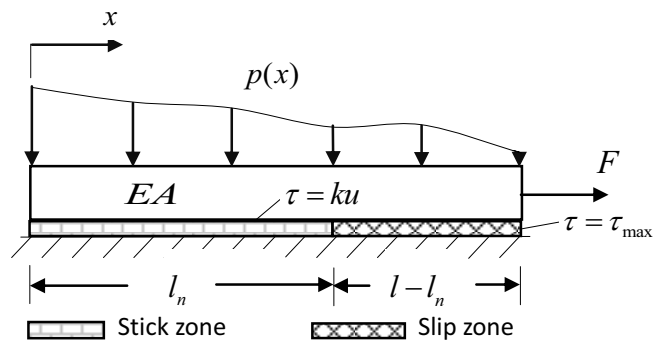

Figure 2: Microslip model of the lap joint.

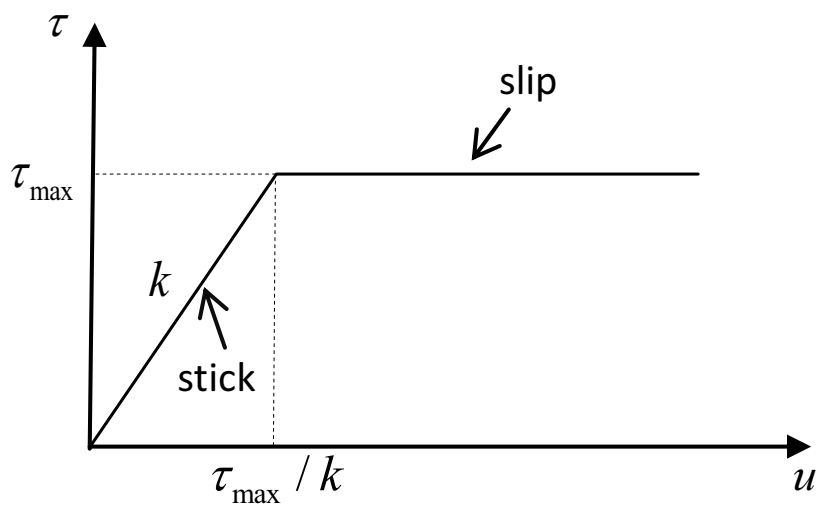

Figure 3: The stress-displacement relationship of the shear layer.

is the displacement at a certain point on the shear layer. The stiffness per unit length of the shear layer in the stick zone is $k . \tau_{\text {max }}$ is the stress of a certain point on the shear layer when it is subjected to slip. $\tau_{\max }=\mu p$, where $\mu$ and $p$ denote the interface friction coefficient and the interface pressure respectively.

In order to express the pressure distribution under equal pre-tension force, the following power function is constructed:

$$
p(x)=c_{\alpha} c_{\beta} p_{0}\left(1-\left(\frac{x}{l}\right)^{\alpha}\right) \quad 0 \leq x \leq l
$$

where, $c_{\alpha}$ is the coefficient of the power function, which is related to the power exponent.

$$
c_{\alpha}= \begin{cases}1 & \alpha=0 \\ \frac{\alpha+1}{\alpha} & \alpha>0\end{cases}
$$

$c_{\beta}$ is the length coefficient, which is related to the length of the contact area. $L=L / \beta$ and $c_{\beta}=\beta$.

$p_{0}$ is the uniform normal pressure value distributed along the length of $L$. Namely, when $\alpha=0$ and $\beta=1$, then $p(x)=p_{0}$.

For generalization, different values are assigned to $\alpha$ and $\beta$ as shown in the formulas below.

$$
p(x)=\left\{\begin{array}{llll}
p_{0} & \alpha=0, \beta=1 & x \leq L & (a) \\
2 p_{0}\left(1-\frac{x}{l}\right) & \alpha=1, \beta=1 & x \leq L & (b) \\
3 p_{0}\left(1-\left(\frac{x}{l}\right)^{2}\right) & \alpha=2, \beta=2 & x \leq \frac{L}{2} & (c) \\
4 p_{0}\left(1-\frac{x}{l}\right) & \alpha=1, \beta=2 & x \leq \frac{L}{2} & (d) \\
6 p_{0}\left(1-\left(\frac{x}{l}\right)^{1 / 2}\right) & \alpha=\frac{1}{2}, \beta=2 & x \leq \frac{L}{2} & (e)
\end{array}\right.
$$

where $(a)$ represents the uniform pressure distribution and also represents the contact pressure at the right-hand end of the overlap zone not yet attenuating to zero. The values of $\alpha$ in $(b)$ and $(d)$ are the same, which represent two modes of distribution with the same power exponent but different contact areas. The values of $\beta$ in $(c),(d)$ and $(e)$ are 


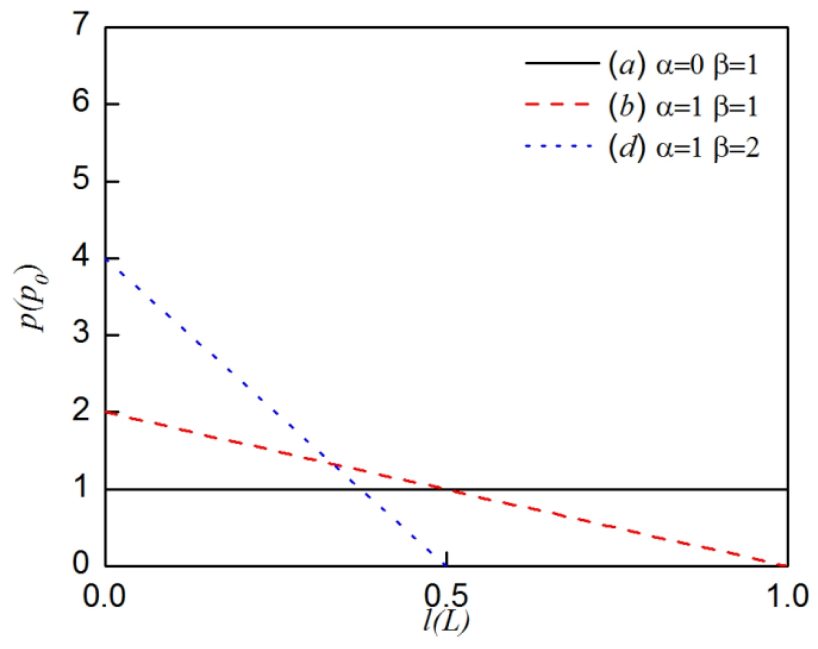

(a) Group one

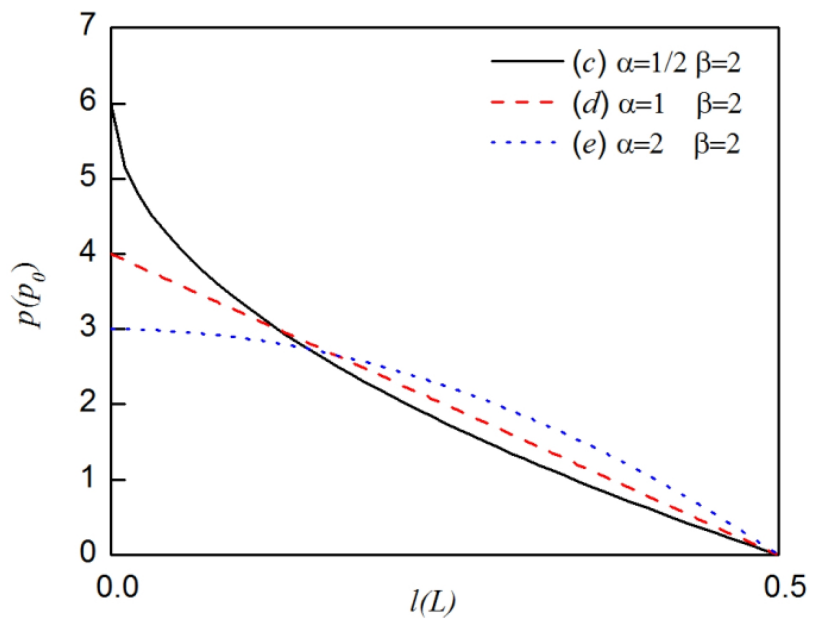

(b) Group two

Figure 4: Pressure distribution graphs.

the same, representing distributions with the same contact area but different power exponents. For comparison, take $(a),(b)$ and $(d)$ as group one and $(c),(d)$ and $(e)$ as group two. Figure 4 shows the plots of the two groups.

The right end of the elastic rod is subject to a tangential load, $F$. The shear layer is divided into a stick zone and a slip zone. We assume the stick zone extends from $x=0$ to some point $x=l_{n}$ (where $l_{n}$ is the length of the stick zone) and the region beyond that point constitutes the slip zone. The displacement of all points along the $x$ direction can be calculated.

\section{Calculation of the microslip responses}

The governing equations defining the microslip situation are defined as

For the stick zone:

$$
E A u^{\prime \prime}(x)-k u(x)=0 \quad 0 \leq x \leq l_{n}
$$

For slip zone:

$$
E A u^{\prime \prime}(x)-\mu p(x)=0 \quad l_{n} \leq x \leq l
$$

The boundary conditions at both ends of the rod are

$$
E A u^{\prime}(0)=0, \quad E A u^{\prime}(l)=F
$$

The continuity condition at $x=\ln$ must be satisfied

$$
u\left(l_{n}\right)^{-}=u\left(l_{n}\right)^{+}, \quad u^{\prime}\left(l_{n}\right)^{-}=u^{\prime}\left(l_{n}\right)^{+}
$$

The superscripts + and - denote limiting values from right and left of transition point $\left(x=l_{n}\right)$ respectively.

Solving equation (2) and (3) with boundary and continuity conditions leads to the displacement at a point distance $x$ from the left end of the beam.

When $\alpha \neq 0$,

$$
u(x)= \begin{cases}\frac{\left[F-\mu p_{0}\left(l-l_{n}\right)\right] \cosh (\gamma x)}{E A \gamma \sinh \left(\gamma l_{n}\right)} & 0 \leq x<l_{n} \\ D_{2} x^{2}+D_{1} x+D_{0} & l_{n} \leq x \leq l\end{cases}
$$

Where,

$$
\begin{gathered}
D_{0}=\frac{F-p_{0} \mu\left(l-l_{n}\right)}{E A \gamma \tanh \left(\gamma l_{n}\right)}-\left[\frac{p_{0} \mu l_{n}^{2}}{2 E A}+\frac{\left(F-p_{0} \mu l\right) l_{n}}{E A}\right], \\
D_{1}=\frac{F+P_{0} \mu l}{E A}, D_{2}=\frac{p_{0} \mu}{2 E A}, \gamma=\sqrt{\frac{k}{E A}} .
\end{gathered}
$$

When $\alpha>0$,

$$
u(x)= \begin{cases}\frac{\cosh (\gamma x)}{\gamma \sinh \left(\gamma l_{n}\right)} \cdot\left(-\frac{C}{L^{\alpha}} \cdot \frac{1}{\alpha+1} \cdot l_{n}^{\alpha+1}\right. & \\ \left.+C l_{n}+\frac{F}{E A}-\frac{\alpha C l}{\alpha+1}\right) & 0 \leq x<l_{n} \\ D_{3}^{\star} x^{\alpha+2}+D_{2}^{\star} x^{2}+D_{1}^{\star} x+D_{0}^{\star} & l_{n} \leq x \leq l\end{cases}
$$

Where,

$$
\begin{aligned}
D_{0}^{\star} & =\frac{1}{\gamma \tanh \left(\gamma l_{n}\right)}\left(-\frac{C}{l^{\alpha}(\alpha+1)} \cdot l_{n}^{\alpha+1}+C l_{n}+\frac{F}{E A}-\frac{\alpha C l}{\alpha+1}\right) \\
& -\left[-\frac{C l_{n}^{\alpha+2}}{l^{\alpha}(\alpha+1)(\alpha+2)}+\frac{C l_{n}^{2}}{2}+\frac{F l_{n}}{E A}-\frac{\alpha C l l_{n}}{\alpha+1}\right] \\
D_{1}^{\star} & =\frac{F}{E A}-C l \cdot \frac{\alpha}{\alpha+1}, \quad D_{2}^{\star}=\frac{C}{2}, \\
D_{3}^{\star} & =-\frac{C}{l^{\alpha}} \cdot \frac{1}{(\alpha+1)(\alpha+2)}, \quad C=\frac{c_{\alpha} c_{\beta} p_{0} \mu}{E A}
\end{aligned}
$$




\section{Effect of interface pressure distributions on stick-slip transitions}

As mentioned previously, the microslip is the main mechanism for the energy dissipation of the joint interface. When analyzing the energy dissipation associated with the microslip, we should first determine the slip zone. Therefore, investigating the effect of interface characteristics on the stick-slip transitions forms the premise for analyzing how it affects the energy dissipation.

There is a stress-continuity condition at the critical point $x=l_{n}$.

$$
k u\left(l_{n}\right)^{-}=\mu p\left(l_{n}\right)^{+}
$$

When $\alpha=0$, combining equations (1) and (6) yields

$$
F=\frac{E A p_{0} \mu \gamma \tanh \left(\gamma l_{n}\right)}{k}+p_{0} \mu\left(l-l_{n}\right)
$$

When $\alpha>0$, combining equations (1) and (7) yields

$$
\begin{aligned}
F & =\frac{E A p\left(l_{n}\right) \mu \gamma \tanh \left(\gamma l_{n}\right)}{k}+\frac{E A C}{l^{\alpha}} \cdot \frac{l_{n}^{\alpha+1}}{\alpha+1} \\
& +\frac{E A \alpha C l}{\alpha+1}-E A C l_{n}
\end{aligned}
$$

Equations (9) and (10) represent the nonlinear relationship between the length of the stick zone length $l_{n}$ and the force $F$.

If $l_{n}=l$, then the minimum force required to start a microslip can be determined.

When $\alpha=0$,

$$
F_{\min }=\frac{E A p_{0} \mu \gamma \tanh (\gamma l)}{k}
$$

As it can be seen that the minimum load is proportional to $p_{0}$.

When $\alpha>0$,

$$
F_{\text {min }}=0
$$

That is, if the contact pressure attenuates to zero within the overlap zone, a microslip starts while load is applied.

When $l_{n}=0$, the load inducing the macroslip can be determined.

$$
F_{\max }=c_{\beta} l p_{0} \mu=L p_{0} \mu
$$

The tangential force required to start a microslip is related to the pre-tension force, i.e. $L p_{0}$ As the pre-tension forces for the five pressure distributions are the same, so the tangential forces to start macroslips are the same too.

Figure 5 shows instances of stick-slip transitions under tangential loads for the two groups of pressure distributions. Throughout this paper, we take $E=2 \times 10^{11} \mathrm{MPa}$,

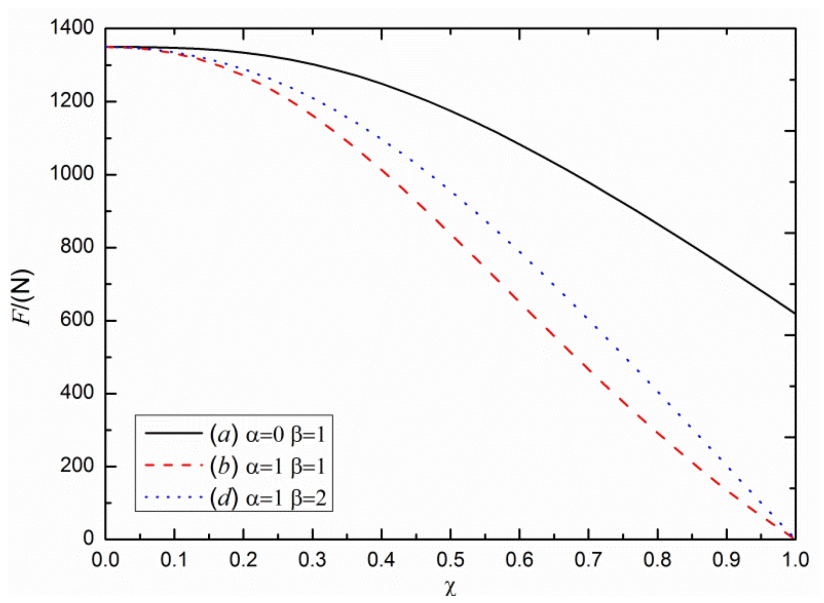

(a) Group one

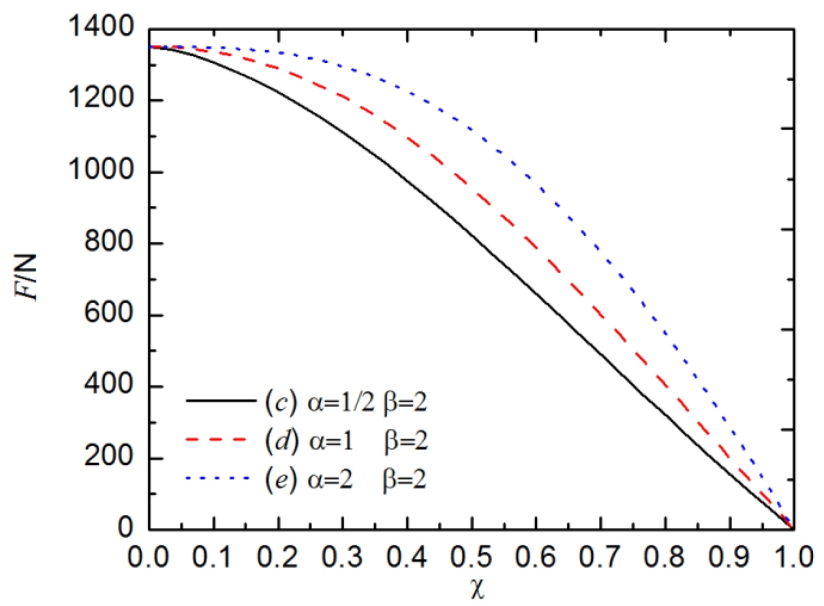

(b) Group two

Figure 5: Instances of the relationship between the length of the stick zone and tangential force.

$A=1 \mathrm{~mm}^{2}, L=30 \mathrm{~mm}, p_{0}=150 \mathrm{MPa}, k=10 \mathrm{GPa}$ and $\mu=0.3$.

For comparison, the length of the stick zone is normalized as $\chi$, i.e. $\chi=l_{n} l$. In figure 5 , the horizontal axis is $\chi$. The vertical axis is the tangential load $F$. Each curve represents a critical curve of the stick-slip transition on which the left side corresponds to the stick zone and the right side corresponds to the slip zone. Each critical curve starts on the left side, which indicates initialization of the microslip, and is the stick-microslip transition point. The right end point, which indicates the start of the macroslip, is the microslipmacroslip transition point.

It is obvious that the distributions affect the microslips of the interfaces. For group one, distribution $(a)$ needs a certain force, i.e. $F=618 \mathrm{~N}$ in this instance, to start the microslip. Before that, the interface is completely stuck. For (b) and $(d)$, only the value of $\beta$ is different. It shows that the 
normalized length of the slip zone is relatively large when $\beta=1$ under the same force, except for the start point and the end point. For distribution $(a)$, the normalized length of the slip zone is always less than that of $(b)$ and $(d)$, because its minimum normal pressure of the overlapped area is non-zero.

For group two, only the power exponents are different. Under the same tangential forces, distribution $(c)$, i.e. $\alpha=1 / 2$, has the largest length of the slip zone. Distribution (e), i.e. $\alpha=2$, has the smallest one. That is to say, for group two, the length of the slip zone is negatively correlated with the exponents. This is mainly because, if $\alpha$ is relatively small, the pressure of the right-hand area of the elastic rod is smaller and the yield stress is smaller as well. Thus microslip is more likely to happen.

\section{Effect of pressure distributions on the hysteretic characteristics}

The interface stick-slip transition results in forcedisplacement hysteresis. The hysteretic loop formed by force and displacement reflects stiffness softening and energy dissipation of the structure experienced repeatedly during loading process. The force-displacement curve obtained due to a monotonically increasing tangential force is called a hysteretic skeleton curve.

\subsection{The hysteretic skeleton curve}

Let $x$ in formula (6) and (7) be $l$, and the forcedisplacement relationship at the right-hand end of the elastic rod can be obtained as

$$
F=k_{f} u(l)+C_{f}
$$

Where

$$
\begin{aligned}
& k_{f}=\frac{1}{\frac{l-l_{n}}{E A}+\frac{1}{E A \gamma \tanh \left(\gamma l_{n}\right)}}
\end{aligned}
$$

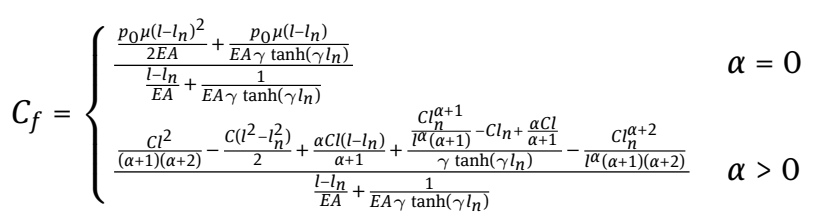

Supposing $l_{n}=l$, for the pressure distribution of $(\alpha)$, i.e. at $\alpha=0$, the model is completely stuck. For other distributions, the microslip starts.

$$
k_{f}=E A \gamma \tanh (\gamma l)
$$

This indicates that initial tangential stiffness of the contact area is related to the length of $l$.

If $l_{n}=0$, the model is in macroslip.

$$
k_{f}=0
$$

Figure 6 shows the skeleton curves for different pressure distributions. From the reduction of the curve slopes with increasing amplitude, we can see the stiffness softening behavior of the model.

For group one, with increasing force, the skeleton curve of distribution $(\alpha)$ is divided into linear, nonlinear and constant sections for stick, microslip and macroslip. The initial slopes of the curves for $(b)$ and $(\alpha)$ are identical, because both have the same contact area, $l$. For distribution $(d)$, the length of the contact area $l=L / 2$. In order to compare $(d)$ with the former two in the length of $L$, a section of $L / 2$ length of elastic rod, not in contact with the

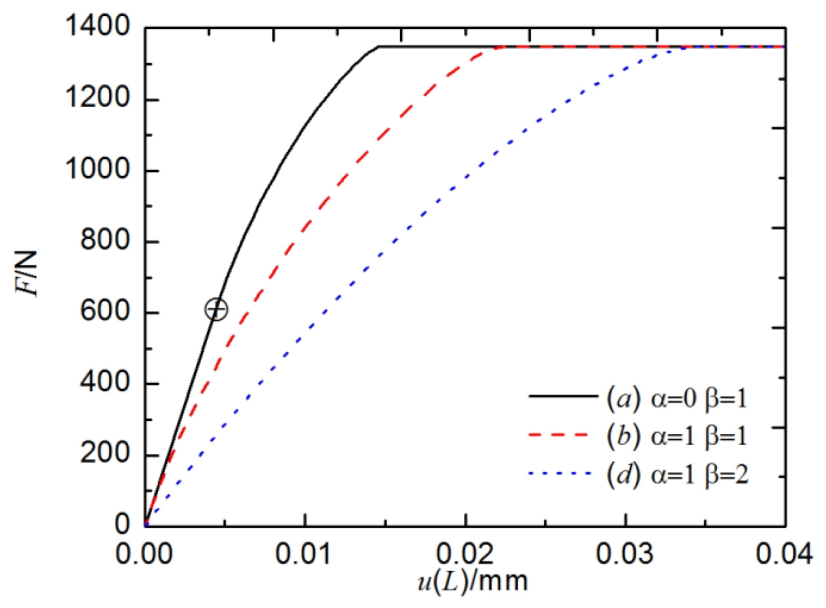

(a) Group one

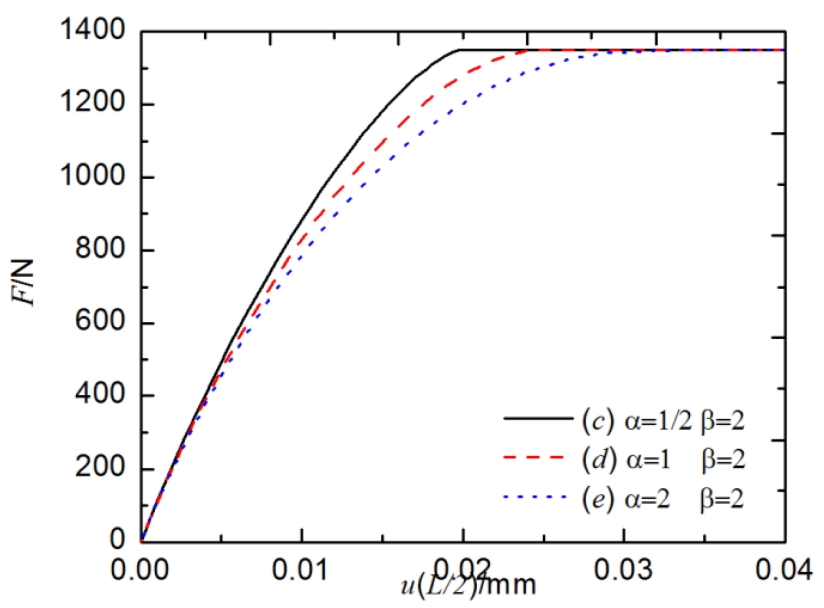

(b) Group two

Figure 6: Skeleton curves for different pressure distributions ( $\oplus$ denotes microslip starting for distribution $(\alpha)$ ). 
rigid base, is added at the right-hand end of the original rod. Because the section of elastic rod has no contact stiffness, the slope of the skeleton curve for $(d)$ is obviously lower than that of $(\alpha)$ and $(b)$.

As predicted, the distributions of group two present the same initial stiffness and their contact areas are the same. With increase of the tangential load, the joint stiffness is positively correlated with $\alpha$. As described in section 4, it is easier to induce microslip at the right-hand side of the elastic rod when $\alpha$ is smaller, which results in a decrease of joint stiffness.

\subsection{The hysteretic loops}

It will be helpful to further understand the dynamic of the interface with the hysteretic loops, which can be obtained based on the skeleton curves and the Masing rule $[18,19]$. The mathematical expression for the unloading can be expressed as

$$
F_{u}=F_{0}-2 k_{f}\left(\frac{u(l)_{0}-u(l)_{u}}{2}\right)-2 C_{f}
$$

Where, $F_{0}$ and $u(L)_{0}$ are the force and displacement values at which the loading process reversed. $F_{u}$ and $u(L)_{u}$ are the unloading force and displacement, respectively. After unloading, the reloading process can be expressed as

$$
F_{r}=-F_{0}+2 k_{f}\left(\frac{u(l)_{0}+u(l)_{u}}{2}\right)+2 C_{f}
$$

Figure 7 shows the hysteretic curves of the microslip model for different pressure distributions. Here, the load amplitudes are chosen to be $800 \mathrm{~N}$. The area of the hysteretic loop represents the amount of energy dissipation per cycle. As it can be seen from the figure, under the chosen force amplitude, the energy dissipation varies with the pressure distribution modes. For group one, the energy dissipation of distribution $(\alpha)$ is the lowest. $(b)$ and $(d)$ have the same power exponent. The distribution which has larger contact area, i.e. (b), dissipates more energy than $(d)$. For group two, it is obvious that the energy dissipation is negatively correlated with power exponent $\alpha$.

The advantage of energy dissipation based on a hysteretic loop is that the loop can be plotted directly by the skeleton curve. There is a certain force amplitude for each loop. It is hard to get the energy dissipation results of the forces from microslip to macroslip. This problem can be solved by using the friction work to calculate the energy dissipation, as shown in the next section.

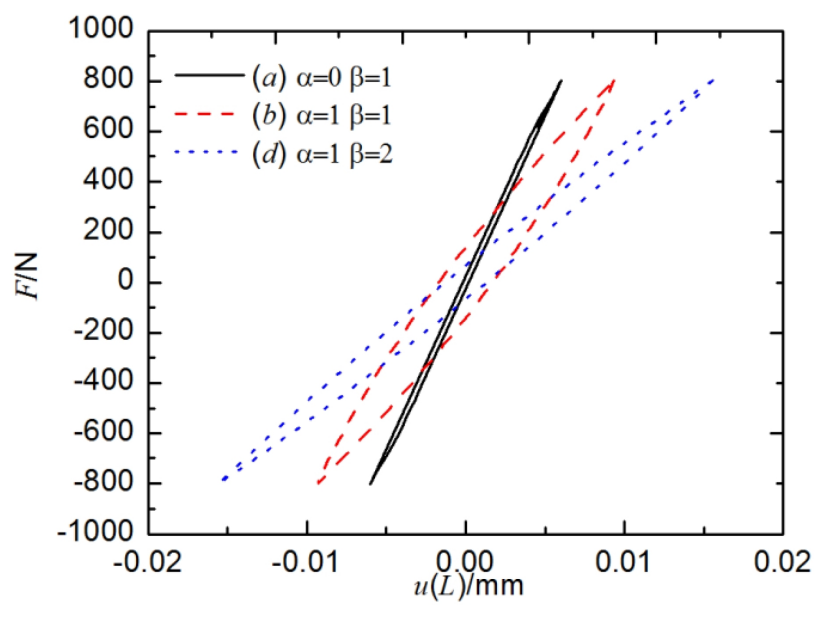

(a) Group one

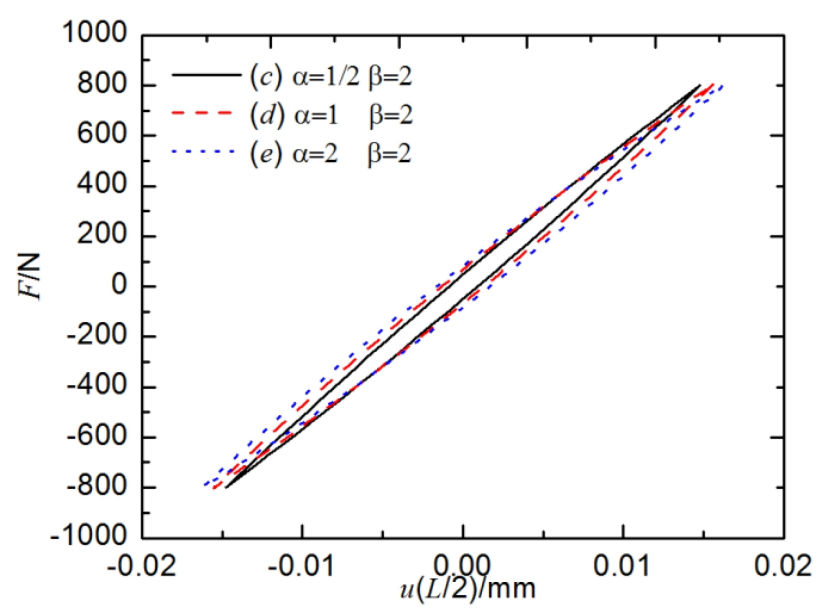

(b) Group two

Figure 7: Hysteretic loops for different pressure distributions.

\section{The effect of pressure distributions on the joint disspation}

The displacement at position $x$ is the sum of the elastic displacement and the slip displacement

$$
u(x)=u_{c}(x)+u_{s}(x)
$$

Where, $u_{c}(x)$ is the critical displacement when point $x$ is transformed from stick to slip, $u_{c}(x)=\mu p(x) / k$. This is the elastic displacement and has no relation with energy dissipation. $u_{s}(x)$ is the slip displacement which will generate energy dissipation. The energy dissipation per cycle gener- 
ated by the interface friction is

$$
\begin{aligned}
\Delta E_{D} & =4 \int_{l_{n}}^{l} \mu p(x) u_{s}(x) \mathrm{d} x \\
& =4 \int_{l_{n}}^{l} \mu p(x)\left[u(x)-u_{c}(x)\right] \mathrm{d} x
\end{aligned}
$$

Therefore, the energy dissipation under different pressure distributions can be obtained.

\subsection{The energy dissipation per cycle on linear coordinates}

Figure 8 shows the energy dissipation per cycle $\Delta E_{D}$ as a function of force amplitudes $F$ for different pressure distributions. From the figure it can be seen that, with increasing force, the energy dissipation increases, and a nonlinear relationship exists.

For the pressure distribution $(\alpha)$ of group one, when the tangential force is small, i.e. $F<618 \mathrm{~N}$ in this instance, the model doesn't produce any dissipation because the interface is completely stuck and there is only elastic displacement. Under the same load, the energy dissipation of $(\alpha)$ is far less than that of distributions $(b)$ and $(d)$. For distribution $(b)$ with a contact area of $L$, and distribution $(d)$ with a contact area of $L / 2$, the former dissipates more energy than the latter. As can be seen from section 4, distribution, ( $b$ ) has a longer length of slip zone and larger slip displacement, so more energy dissipation is produced.

The distributions of group two have the same contact area but different power exponents. At the beginning of microslip, namely when the tangential load is relatively small, it is hard to distinguish the energy dissipation curves of the three. The interface pressures and microslip areas are nearly the same at the beginning of the curves. With increasing load, the differences between the energy curves increase and the energy dissipation capabilities decrease in a sequence of $\alpha=1 / 2, \alpha=1$ and $\alpha=2$.

\subsection{The energy dissipation on log-log coordinates}

A power law relationship exists between the energy dissipation per cycle and the load amplitudes, i.e. $\Delta E_{D}=\alpha F^{n}$, $2 \leq n \leq 3.3[20,21]$. That is to say, on log-log coordinates, a linear relationship exists and the slope of the curve represents the nonlinearity degree between energy dissipation

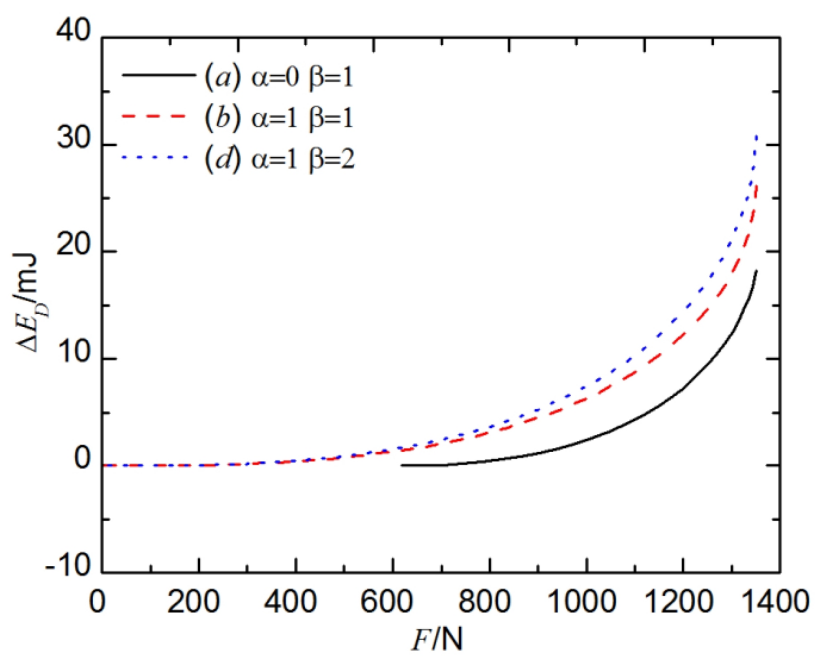

(a) Group one

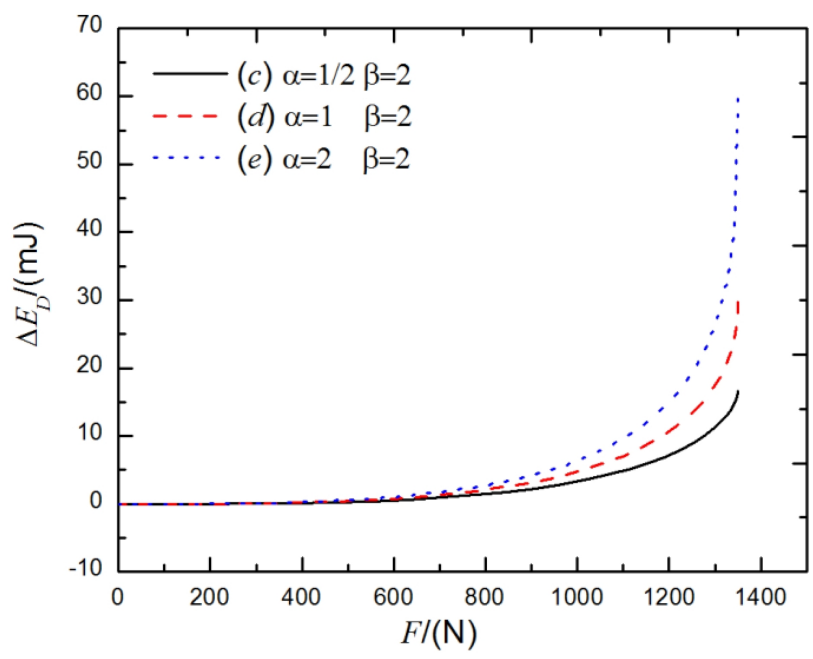

(b) Group two

Figure 8: Instances of energy dissipation per cycle for different pressure distributions.

per cycle and the load amplitudes. This feature is considered to be the main measure for distinguishing the energy dissipation characteristics of different joint interfaces.

Figure 8 is replotted on log-log coordinates in Figure 8. It can be seem that, if the data corresponding to small force amplitudes are ignored, the curve can be approximated to a straight line. The curves are fitted and the slopes of the lines can be obtained, as shown in Table 1 .

As can be seen from the table, the minimum value of the power law exponent $n$ is 2.77 and the maximum value is 3.09, which exhibits similar variation to the experimental results[10]. The power law exponent between the unit periodical energy dissipation and the load amplitude is related to the mode of pressure distribution. To be specific, for the distributions of group one, distribution $(\alpha)$ 
has the strongest nonlinear behavior. For the distributions of group two, it is obvious that, with the increasing values of $\alpha$, the slope of the curves decrease correspondingly; namely, the nonlinear behaviors recede.

Table 1: power-law exponents $n$ between energy dissipation per cycle and the tangential load amplitudes for different pressure distributions

\begin{tabular}{cccc}
\hline Group one & $n$ & Group two & $n$ \\
\hline$(\alpha)=1, \beta=1$ & 3.09 & (c) $\alpha=1 / 2, \beta=2$ & 2.90 \\
$(b) \alpha=1, \beta=1$ & 2.77 & (d) $\alpha=1, \beta=2$ & 2.77 \\
$(d) \alpha=1, \beta=2$ & 2.79 & (e) $\alpha=2, \beta=2$ & 2.63 \\
\hline
\end{tabular}

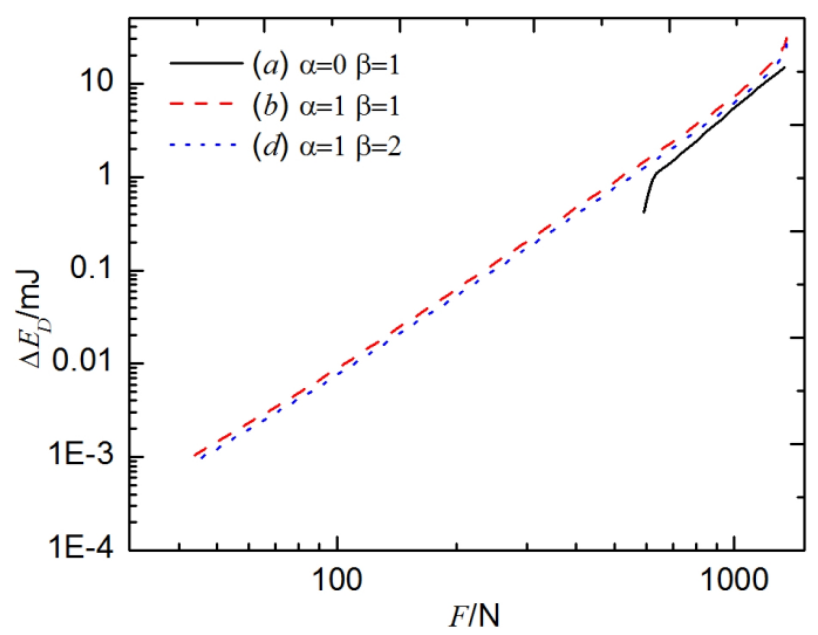

(a) Group one

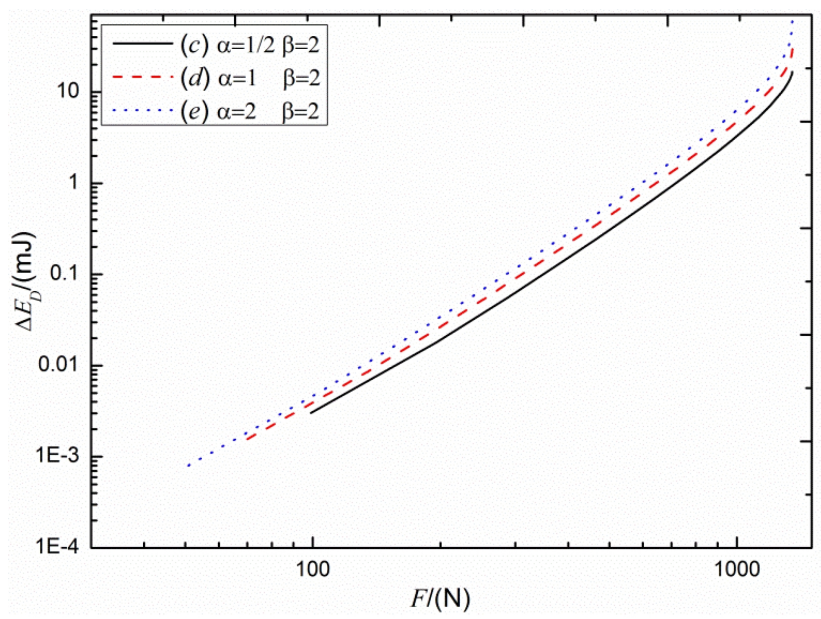

(b) Group two

Figure 9: Log-log plots of energy dissipation for different pressure distributions.

\section{Conclusion}

This paper constructs a microslip model simulating a bolted lap joint. Five contact pressure distributions are considered, all with equal pre-tension force. This paper investigates the effects of different pressure distributions on the interface stick-slip transitions and hysteretic characteristics. As can be seen, both the amount and nonlinearity of the joint energy dissipation are related to factors including: whether the pressure within the overlapped area attenuates to zero, the size of the contact area, and the value of the power exponent. The study theoretically shows that the energy dissipation capability can be improved by reasonably designing the structure to obtain a desired interface pressure distribution, even if the pre-tension force is the same.

Acknowledgement: The work has been supported by PHD Programs Foundation of Ludong University (25970301).

\section{References}

[1] Beards C.F., The damping of structural vibration by controlled interface slip in joints, J. Vib., Acoust., Stress, and Reliab., 1983, 105, 369-373.

[2] Walker S., Aglietti G., Cunningham P., A study of joint damping in metal plates, Acta Astronautica, 2009, 65, 184-191.

[3] Gaul L. , Lenz J., Nonlinear dynamics of structures assembled by bolted joints, Acta Mechanica, 1997, 125, 169-181.

[4] Iranzad M., Ahmadian H., Identification of nonlinear bolted lap joint models, Comput. Struct., 2012, 96-97, 1-8.

[5] Ouyang H. , Oldfield M.J., Mottershead J.E., Experimental and theoretical studies of a bolted joint excited by a torsional dynamic load, Int. J. Mech. Sci., 2006, 48, 1447-1455.

[6] Chatelet E., Michon G., Manin L., Jacquet G., Stick/slip phenomena in dynamics: Choice of contact model, Numerical predictions \& experiments, Mech. Mach. Theory, 2008, 43, 1211-1224.

[7] Ungar E.E., The status of engineering knowledge concerning the damping of built-up structures, J. Sound Vib., 1973, 26, 141-154.

[8] Groper M., Microslip and macroslip in bolted joints, Exp. Mech., 1985, 25, 171-174.

[9] Padmanabhan K. K., Murty A. S. R., Damping in structural joints subjected to tangential loads., Proc. Inst. Mech. Eng., Part C, 1991, 205, 121-129.

[10] Smallwood D.O., Gregory D.L., Coleman R.G., Damping Investigations of a Simplified Frictional Shear Joint. Sandia National Labs., Albuquerque, NM (US); Sandia National Labs., Livermore, CA (US), 2000.

[11] Nanda B.K., Study of the effect of bolt diameter and washer on damping in layered and jointed structures, J. Sound Vib., 2006,290,1290-1314.

[12] Stephen J. T., Marshall M.B., Lewis R., An investigation into contact pressure distribution in bolted joints. Proceedings of the 
Institution of Mechanical Engineers, Part C: Journal of Mechanical Engineering Science, 2014, 228, 3405-3418.

[13] Menq C. H., Bielak J., Griffin J. H., The influence of microslip on vibratory response, part I: a new microslip model, J. Sound Vib., 1986, 107, 279-293.

[14] Csaba G., Forced response analysis in time and frequency domains of a tuned bladed disk with friction dampers, J. Sound Vib., 1998, 214, 395-412.

[15] Song Y., McFarland D.M., Bergman L.A., Effect of pressure distribution on energy dissipation in a mechanical lap joint, AIAA journal, 2005, 43, 420-425.

[16] Cigeroglu E., Lu W., Menq C. H., One-dimensional dynamic microslip friction model. J. Sound Vib., 2006, 292, 881-898.
[17] Xiao H., Shao Y., Xu J., Investigation into the energy dissipation of a lap joint using the one-dimensional microslip friction model, Eur. J. Mech. A-SOLID, 2014, 43, 1-8.

[18] Masing G., Eigenspannungen und Verfestigung beim Messing [Fundamental stresses and strengthening with brass], International Congress of Applied Mechanics, 2d, Zirich, 1926.

[19] Segalman D. J., Starr M. J., Inversion of Masing models via continuous Iwan systems, Int. J. Nonlin. Mech., 2008, 43, 74-80.

[20] Ungar E. E., The status of engineering knowledge concerning the damping of built-up structures., J. Sound Vib., 1973, 26, 141-154.

[21] Hartwigsen C. J., Song Y., McFarland D. M., Bergman L. A., Vakakis A. F., Experimental study of non-linear effects in a typical shear lap joint configuration, J. Sound Vib., 2004, 277, 327-351. 